\title{
Presencia e influencia de modelos profesionales familiares del área de salud en la decisión de estudiar medicina en una cohorte de médicos titulados de la Universidad de Antofagasta
}

\author{
Claudia Álvarez-Iguaín, Natalia Becerra-Mellado, Bárbara Gutiérrez-Pereira, Alberto Torres-Belma, \\ Diego Varas-Varas
}

Introducción. Es importante explorar la presencia e influencia de los modelos profesionales de la familia del médico titulado en su decisión de estudiar medicina.

Objetivo. Determinar la presencia e influencia de los modelos profesionales familiares del área de salud en la decisión de estudiar medicina en una cohorte de médicos titulados.

Sujetos y métodos. Estudio cuantitativo transversal en el que participaron 38 médicos recién titulados de la Universidad de Antofagasta, quienes respondieron la encuesta del programa de retroalimentación curricular de la Unidad de Educación Médica del Departamento de Ciencias Médicas de la Universidad de Antofagasta.

Resultados. La mayoría de los encuestados cuentan con familiares profesionales del área de salud. En relación a la influencia de los modelos profesionales familiares en la elección profesional, los resultados no son categóricos. Se aprecia que quienes logran la titulación oportuna en siete años, refieren en su mayoría contar con profesionales familiares del área de salud y sentirse influidos por dichos modelos al momento de elegir su carrera.

Conclusiones. La influencia en la decisión de estudiar medicina puede atribuirse tanto a motivaciones derivadas de la influencia familiar como de la expresión de la subjetividad del individuo.

Palabras clave. Actitud. Familiares. Intención. Miembros de la familia. Redes de parentesco.

Presence and influence of professional family models of the health area in the decision to study medicine in a cohort of medical graduates from the University of Antofagasta

Introduction. It is important to explore the presence and influence of the professional models of the family of the qualified physician in their decision to study Medicine.

Aim. To determine the presence and influence of professional family models in the health area in the decision to study medicine in a cohort of medical graduates.

Subjects and methods. A cross-sectional quantitative study involving 38 recently graduated doctors from the University of Antofagasta, who answered the Survey of the Curricular Feedback Program of the Medical Education Unit of the Department of Medical Sciences of the University of Antofagasta.

Results. Most of the respondents have professional family members of the health area. In relation to the influence of professional family models in professional choice, the results are not categorical. It is appreciated that those who achieve the appropriate degree in seven years, mostly refer to family professionals in the health area and feel influenced by these models when choosing their career.

Conclusions. The influence on the decision to study medicine can be attributed both to motivations derived from the family influence and the expression of the subjectivity of the individual.

Key words. Attitude. Family. Intention. Kinship networks. Members of the family.

\section{Introducción}

En el proceso de elección de una carrera universitaria pueden influir múltiples factores, entre ellos, diferentes motivaciones que a su vez configuran diversos tipos de decisión y cursos de acción.
Según Blustein, citado por Basterretche y Carrasco, las motivaciones para elegir una carrera son intrínsecas y extrínsecas: la motivación intrínseca depende de la necesidad de realizar actividades que brinden satisfacción; las extrínsecas, de factores externos como la influencia familiar o de grupo [1].
Departamento de Ciencias Médicas. Facultad de Medicina y Odontología. Universidad de Antofagasta. Antofagasta, Chile.

Correspondencia:

Dr. Alberto Torres Belma. Departamento de Ciencias Médicas. Facultad de Medicina y Odontología. Universidad de Antofagasta. Avda. Argentina, 2000. Antofagasta, Chile.

E-mail:

alberto.torres@uantof.cl

Recibido:

05.07.18.

Aceptado:

18.07.18.

Conflicto de intereses: No declarado.

Competing interests: None declared.

(c) 2018 FEM 
Lo anterior se complementa con los factores condicionantes de la elección de una carrera. En ese sentido, Osipow, citado por Rivas, plantea que existen distintos factores, entre ellos: hogar y familia, personalidad, realización, intereses profesionales... [2].

Desde el punto de vista de la sociología, esto puede derivar en diversos tipos de acciones para concretar la acción de estudiar una profesión, entre ellas, la acción tradicional y la acción racional con arreglo a valores. Según Weber, citado por Giner, la acción tradicional se relaciona con un hábito incorporado y costumbres establecidas, muchas veces una mera reacción rutinaria a un estímulo no individual, que está en el límite de la acción con sentido subjetivo. Por su parte, la acción racional con arreglo a valores es siempre una acción según precepto o de acuerdo con una exigencia que el actor cree que le está dirigida [3].

En el caso de la acción tradicional encontramos el patrón de conducta según el cual el futuro estudiante escoge su profesión en función de modelos familiares presentes, que influyen fuertemente en su decisión. En la acción racional con arreglo a valores prevalecería el juicio individual que inspiraría la elección de la profesión, dando lugar a la plena expresión de la subjetividad, la cual podría verse inspirada por diversos motivos, como la necesidad de desarrollar acciones de prevención y promoción de la salud en la comunidad en la que desempeña su futuro profesional.

Según Ferreyra y Pedrazzi [4], desde la perspectiva de la psicología del aprendizaje, es posible abordar este fenómeno, citando a Albert Bandura y su aprendizaje observacional. Este se considera como una forma de aprendizaje en que el observador efectúa la conexión entre la conducta y sus consecuencias, sin necesidad de pasar por la vivencia directa de éstas. Además, dentro del aprendizaje observacional, Bandura distingue cuatro factores fundamentales: atención, retención de lo observado, producción y motivación. Dentro de este último punto, encontramos tres fuentes básicas de la motivación: pensamiento y proyecciones, establecer metas y autosuficiencia. Este último concepto es muy importante en la teoría de Bandura (y sobre todo en la actual investigación) y se refiere a nuestras creencias acerca de nuestra aptitud personal en un área determinada.

Vásquez [5], en relación a la perspectiva de Bandura, sostiene que gran parte del aprendizaje de la conducta social se logra observando las conductas de otros y este aprendizaje se denomina 'aprendizaje por observación'. La conducta imitativa puede resultar de la observación de un modelo. En esta frase podemos encontrar resumidos los tres conceptos más importantes del aprendizaje por imitación: 'observar', 'imitar' y 'modelos'.

Considerando los resultados de un estudio, realizado en España, en el cual se realizaron encuestas a 105 alumnos correspondiente al segundo curso de la carrera de medicina, del Departamento de Farmacología y Fisiología de la Facultad de Medicina de Zaragoza, se destaca lo siguiente: un $60 \%$ de los estudiantes pensaron en estudiar medicina antes de los 14 años, incluso un 38\% antes de los 10 años. En cuanto a los factores influyentes en la decisión de estudiar medicina, un 70\% declaró la existencia de algún factor influyente en su decisión; de este grupo, la mayoría contestó que el principal factor influyente era la presión familiar (16\%), en segundo lugar, el contacto con la enfermedad (15\%) y, posteriormente, la existencia de familiares médicos en el entorno (12\%). Este dato permite señalar cierta influencia de familiares con profesiones del área de salud en la decisión de estudiar medicina, aunque la pregunta se orienta a consultar únicamente por la existencia de médicos [6].

En ese sentido, nuestra experiencia pretende determinar la presencia e influencia de modelos profesionales familiares del área de salud en la decisión de estudiar medicina en una cohorte de médicos titulados, a través de un análisis descriptivo. Además, se pretende generar una lectura teórica que permita interpretar los resultados, integrando perspectivas multidisciplinares, citadas en la introducción.

\section{Sujetos y métodos}

Se realizó un estudio cuantitativo transversal de carácter descriptivo en el que participaron 38 médicos recién titulados de la Universidad de Antofagasta, licenciados en 2017, los cuales respondieron el cuestionario del programa de retroalimentación curricular de la Unidad de Educación Médica del Departamento de Ciencias Médicas de la universidad. Se les consultó sobre la existencia e influencia de modelos profesionales familiares del área de salud en la decisión de estudiar medicina y se recopilaron datos de contacto para la actualización de las bases de datos de cohortes egresadas. Por tanto, no se seleccionó una muestra específica, ya que se encuestó a la cohorte licenciada en 2017 (a excepción de una licenciada que no pudo participar en el proceso de recogida de información y de aquellos que no aprobaron el Examen Único Nacional de Conocimientos de Medicina, por la dificultad para acceder a la recopilación de datos). 
Los participantes de la medición fueron $19 \mathrm{mu}$ jeres y 19 hombres, con un rango de edad de 24-30 años, y la mayoría entre 24 y 25 años (64,8\%).

El procedimiento escogido para la recogida de información consistió en una encuesta descriptiva. Sin embargo, asumió rasgos de una encuesta explicativa, ya que facilitó el cruce de dos variables para el análisis de los datos. El instrumento de recogida de información consistió en un cuestionario que consultó sobre dimensiones relativas a datos de contacto, caracterización sociodemográfica del encuestado, opiniones relativas al proceso formativo y existencia de profesionales del área de salud en su respectiva familia (nuclear o extensa) y la posible influencia de estos modelos profesionales en la elección de la carrera de medicina.

Previamente a la aplicación del cuestionario se les informó sobre la necesidad de contar con datos relevantes de cada uno para entablar contacto en el futuro y su acuerdo o desacuerdo al respecto en el momento de responder la encuesta. No se registraron negativas en este punto. Además, la encuesta se aplicó en un tiempo único (un día determinado).

El diseño del estudio corresponde al de investigación no experimental, considerando que la medición se realiza después de ocurridos los hechos. Los resultados de la medición se tabularon y analizaron con el programa estadístico SPSS v. 15.0.

Se realizó un análisis descriptivo, en el cual se mostraron los porcentajes obtenidos en las preguntas planteadas. Además, se emplearon tablas de contingencia para el cruce de aquellas variables que resultaron claves en el análisis.

\section{Resultados}

Casi dos tercios de los egresados (57,9\%) cuentan con familiares cuyas profesiones se incluyen en el área de salud. Consultados respecto a qué profesión del área de salud se halla en sus respectivas familias, la profesión que lidera las menciones, con un $18,4 \%$, es 'enfermero (a)', seguido de 'médico (a)', con un $15,8 \%$. En tercer lugar se ubica 'paramédico (a)', con un 13,2\%, y mucho más atrás, en último lugar, se sitúa la opción 'matrón (a)', con un 5,3\%.

Respecto a la afirmación 'la existencia de profesionales del área de salud en su círculo familiar inspiró la decisión de estudiar medicina, un 36,4\% de los egresados señala estar 'de acuerdo' con ella, y un $27,3 \%$, 'totalmente en desacuerdo'. Según ello, existiría una respuesta favorable al enunciado, pero al sumar los porcentajes de las categorías de respuestas afirmativas ('totalmente de acuerdo' y 'de acuer-
Tabla I. En tu familia, ¿existen profesionales del área de salud?

\begin{tabular}{|c|c|c|c|c|c|c|}
\hline & & \multicolumn{4}{|c|}{ Año de ingreso en la carrera ${ }^{a}$} & \multirow{2}{*}{ Total } \\
\hline & & 2008 & 2009 & 2010 & 2011 & \\
\hline \multirow{2}{*}{ Sí } & $n$ & 2 & 2 & 1 & 17 & 22 \\
\hline & $\%$ & $100 \%$ & $33,3 \%$ & $33,3 \%$ & $63 \%$ & $57,9 \%$ \\
\hline \multirow{2}{*}{ No } & $n$ & 0 & 4 & 2 & 10 & 16 \\
\hline & $\%$ & $0 \%$ & $66,7 \%$ & $66,7 \%$ & $37 \%$ & $42,1 \%$ \\
\hline \multirow{2}{*}{ Total } & $n$ & 2 & 6 & 3 & 27 & 38 \\
\hline & $\%$ & $100 \%$ & $100 \%$ & $100 \%$ & $100 \%$ & $100 \%$ \\
\hline
\end{tabular}

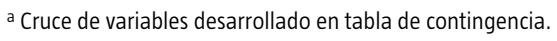

do'), por un lado, y de las categorías de respuesta negativas ('totalmente en desacuerdo' y 'en desacuerdo'), por otro, concluimos que un $50 \%$ responde positiva o negativamente a la afirmación planteada en la encuesta. Los resultados no son categóricos, ya que prevalecen dos realidades contrapuestas.

Los licenciados de medicina de la cohorte de 2011 (titulados oportunamente en 2017) refieren poseer familiares del área de salud en un $63 \%$. Sin embargo, a medida que descienden las cohortes (y por ende, disminuye la titulación oportuna), aumenta el porcentaje de la categoría de respuesta negativa, a excepción de la cohorte de 2008 (Tabla I).

Se construyó una tabla de contingencia para establecer un cruce entre las preguntas 'año de ingreso en la carrera' y 'la existencia de profesionales del área de salud en tu círculo familiar inspiró la decisión de estudiar medicina' (Tabla II). Los resultados del cruce de variables resultan interesantes porque un $35,3 \%$ de los licenciados que ingresaron en 2011 refieren estar 'de acuerdo' con que la existencia de profesionales del área de salud en su familia inspiró su decisión de estudiar medicina, conclusión que también se refleja en el resultado de la cohorte de 2010.

La cohorte de 2010 titulada en 2017 (es decir, con un año de retraso académico) replica la situación anterior porque un $100 \%$ de ellos refiere estar 'de acuerdo' con que la existencia de profesionales del área de salud en sus respectivas familias representó una inspiración a la hora de estudiar medicina.

\section{Discusión}

La investigación permite concluir que la influencia 
Tabla II. La existencia de profesionales del área de salud en tu círculo familiar inspiró la decisión de estudiar medicina.

\begin{tabular}{|c|c|c|c|c|c|c|}
\hline & & \multicolumn{4}{|c|}{ Año de ingreso en la carrera ${ }^{a}$} & \multirow{2}{*}{ Total } \\
\hline & & 2008 & 2009 & 2010 & 2011 & \\
\hline \multirow{2}{*}{$\begin{array}{l}\text { Totalmente } \\
\text { de acuerdo }\end{array}$} & $n$ & 0 & 0 & 0 & 3 & 3 \\
\hline & $\%$ & $0 \%$ & $0 \%$ & $0 \%$ & $17,6 \%$ & $13,6 \%$ \\
\hline \multirow{2}{*}{ De acuerdo } & $n$ & 1 & 0 & 1 & 6 & 8 \\
\hline & $\%$ & $50 \%$ & $0 \%$ & $100 \%$ & $35,3 \%$ & $36,4 \%$ \\
\hline \multirow{2}{*}{ En desacuerdo } & $n$ & 1 & 1 & 0 & 3 & 5 \\
\hline & $\%$ & $50 \%$ & $50 \%$ & $0 \%$ & $17,6 \%$ & $22,7 \%$ \\
\hline \multirow{2}{*}{$\begin{array}{l}\text { Totalmente } \\
\text { en desacuerdo }\end{array}$} & $n$ & 0 & 1 & 0 & 5 & 6 \\
\hline & $\%$ & $0 \%$ & $50 \%$ & $0 \%$ & $29,4 \%$ & $27,3 \%$ \\
\hline \multirow{2}{*}{ Total } & $n$ & 2 & 2 & 1 & 17 & 22 \\
\hline & $\%$ & $100 \%$ & $100 \%$ & $100 \%$ & $100 \%$ & $100 \%$ \\
\hline
\end{tabular}

a Cruce de variables desarrollado en tabla de contingencia.

de modelos familiares del área de salud en la decisión de estudiar medicina de parte de los egresados de la cohorte de 2017 puede atribuirse tanto a una motivación intrínseca como extrínseca, siguiendo la conceptualización de Blustein. Por lo tanto, ninguna de las motivaciones es categórica en su presencia, según se desprende de los datos estadísticos, en donde se aprecia una distribución igual de porcentajes $(50 \%)$ cuando se consulta sobre la influencia de los modelos profesionales familiares en la elección de la profesión médica.

Siguiendo a Osipow, se concluye que existirían distintos factores influyentes en la decisión de estudiar medicina, entre ellos el hogar y la familia, pero también la realización y los intereses profesionales particulares [2].

Desde el punto de vista de la sociología posiblemente influiría una acción de tipo tradicional (replicación del modelo), como también los intereses subjetivos, representados en una acción racional con arreglo a valores, que refleja la expresión de juicios individuales que inspiran la decisión de estudiar medicina.

Por otra parte, de acuerdo con el concepto de aprendizaje observacional de Bandura [4], los modelos familiares del área de salud logran influir en la elección de la carrera de medicina, aunque no de manera absoluta. Además, intervienen factores co- mo la motivación, lo que conlleva establecer metas y autosuficiencia (según Bandura), evidenciadas en la titulación oportuna de los encuestados; por tanto, lo aprendido por medio de la observación desarrolla las aptitudes personales hacia un área profesional determinada, lo que es reforzado por la perspectiva de Vásquez, según la cual la imitación de algún modelo o conducta se logra con la observación de las conductas de otros.

Respecto a la tabla I, la presencia de familiares del área de salud en la familia puede constituir un factor explicativo que facilite el avance curricular de los estudiantes de medicina, asegurando una titulación oportuna.

Finalmente, en relación a la tabla II, los resultados del cruce de variables resultan interesantes porque un $35,3 \%$ de los licenciados que ingresaron en 2011 refieren estar 'de acuerdo' con que la existencia de profesionales del área de salud en su familia inspiró su decisión de estudiar medicina, lo que coincide con una titulación oportuna de dicha cohorte, licenciada en 2017. Esta afirmación se fortalece al sumar el 35,3\% de la categoría 'de acuerdo' con el $17,5 \%$ de la categoría 'totalmente de acuerdo' de la cohorte de 2011, un factor que podría ser un aliciente en la titulación oportuna de los egresados. Esta conclusión también se refleja en el resultado de la cohorte de 2010.

Una limitación del presente estudio es que los encuestados son licenciados que lograron aprobar el Examen Único Nacional de Conocimientos de Medicina. En ese contexto, hubiera sido interesante incluir la visión de médicos titulados de la cohorte de 2017 que no lograron aprobar el examen, aunque el número fue reducido (5-6 licenciados). Ello se atribuye a que la encuesta se aplica cuando aquellos que aprobaron el examen recogen su puntuación en dependencias del Departamento de Ciencias Médicas, instancia en la cual no participan los suspendidos. Por lo tanto, dicha limitación constituye una oportunidad para mejorar una futura aplicación del instrumento.

\section{Bibliografía}

1. Basterretche J, Carrasco J. Técnicas y recursos para motivar a los alumnos. Madrid: Rialp; 2004.

2. Rivas F. La elección de estudios universitarios. Madrid: Impresiones Fareso; 1990.

3. Giner S. Teoría sociológica clásica. Barcelona: Ariel; 2011.

4. Ferreyra H, Pedrazzi G. Teorías y enfoques psicoeducativos del aprendizaje. Buenos Aires: Ediciones Novedades Educativas de México; 2007.

5. Vásquez E. Principios y técnicas de educación de adultos. Costa Rica: Editorial Universidad Estatal a Distancia; 2005.

6. Soria M, Guerra M, Giménez I, Escanero JF. La decisión de estudiar medicina: características. Educ Med 2006; 9: 91-7. 